\title{
Fitting the Gompertz equation to asymmetric breakthrough curves
}

\author{
Khim Hoong Chu \\ Honeychem, Nanjing Chemical Industry Park, Nanjing 210047, China
}

\section{A R T I C L E I N F O}

\section{Keywords:}

Adsorption

Breakthrough curve

Fixed bed

Gompertz

Logistic

\begin{abstract}
A B S T R A C T
Fixed bed adsorption studies often report asymmetric breakthrough curves which exhibit a tailing phenomenon as the effluent approaches the influent concentration. Evaluations of models capable of describing such curves are lacking in the literature. This paper examines the ability of the Gompertz equation to correlate asymmetric breakthrough data collated from reports published in the environmental adsorption literature. It is shown that the Gompertz equation, which has received little attention in this field of research, is able to track asymmetric breakthrough curves displaying a moderate degree of tailing. The logistic equation, which is mathematically analogous to the Bohart-Adams, Thomas, and Yoon-Nelson models, cannot effectively describe such asymmetric data. The Gompertz equation provides only an approximate representation of breakthrough data exhibiting a pronounced degree of tailing. To fit such data, this paper presents two modified forms of the Gompertz equation, which are shown to be highly accurate $\left(R^{2}>0.996\right)$. The Gompertz equation and the two modified versions are useful additions to the toolbox of breakthrough curve modeling which has long been filled with the BohartAdams, Thomas, and Yoon-Nelson models. These popular logistic-based equations are confined to fitting symmetric breakthrough curves.
\end{abstract}

\section{Introduction}

Fixed bed adsorption is an important method for removing organic and inorganic contaminants from drinking water sources and wastewater streams. Several textbooks offer extensive theoretical descriptions of the breakthrough dynamics of fixed bed adsorption columns. They are a valuable source of much information on a wide variety of fixed bed models based on sophisticated mass transfer principles as well as simple chemical rate laws [1-4]. In environmental adsorption research simple models such as the Bohart-Adams, Thomas, and YoonNelson equations are frequently used by investigators to correlate fixed bed breakthrough data. The commonly used linear forms of the three models are written as

Bohart-Adams: $\ln \left(\frac{C_{\mathrm{o}}}{C}-1\right)=\frac{k_{\mathrm{BA}} N_{\mathrm{o}} L}{u}-k_{\mathrm{BA}} C_{\mathrm{o}} t$

Thomas: $\ln \left(\frac{C_{\mathrm{o}}}{C}-1\right)=\frac{k_{\mathrm{T}} q_{\mathrm{o}} M}{Q}-k_{\mathrm{T}} C_{\mathrm{o}} t$

Yoon-Nelson: $\ln \left(\frac{C_{\mathrm{o}}}{C}-1\right)=k_{\mathrm{YN}} \tau-k_{\mathrm{YN}} t$

where the symbols used are defined at the beginning of this paper.

The three models should not be treated separately (as they commonly are) because mathematically they are equivalent to one another.
Each model can be expressed in terms of the logistic equation formulated for adsorption [5-11]:

$\frac{C}{C_{\mathrm{o}}}=\frac{1}{1+\exp (a-b t)}$

where the general parameters $a$ and $b$ are given by

Bohart-Adams: $a=\frac{k_{\mathrm{BA}} N_{\mathrm{o}} L}{u} ; b=k_{\mathrm{BA}} C_{\mathrm{o}}$

Thomas: $a=\frac{k_{\mathrm{T}} q_{\mathrm{o}} M}{Q} ; b=k_{\mathrm{T}} C_{\mathrm{o}}$

Yoon-Nelson: $a=k_{\mathrm{YN}} \tau ; b=k_{\mathrm{YN}}$

Clearly, the three models are exactly analogous. This in turn means that they can be represented by the logistic equation in data fitting, as will be done here. It should however be noted that this mathematical equivalence is sheer algebraic coincidence because there is no intrinsic relation between the three models and the logistic equation.

The logistic function was first propounded by Pierre-François Verhulst in 1838 [12] as a means of describing dynamic population growth in the presence of restricted resources. It has since then been applied to a large number of phenomena that exhibit sigmoidal or Sshaped patterns. However, a major drawback to the use of the logistic equation lies in the fact that it predicts symmetric sigmoidal curves. It

E-mail address: khimchu@gmail.com. 


$\begin{array}{ll}\text { Nomenclature } \\ \text { Roman letters } \\ a & \text { parameter of the logistic equation } \\ b & \text { parameter of the logistic equation } \\ C & \text { outlet adsorbate concentration at time } t \\ C_{\mathrm{o}} & \text { inlet adsorbate concentration } \\ J & \text { number of competing models } \\ k_{\mathrm{BA}} & \text { Bohart-Adams rate coefficient } \\ k_{\mathrm{T}} & \text { Thomas rate coefficient } \\ k_{\mathrm{YN}} & \text { Yoon-Nelson rate coefficient } \\ L & \text { bed depth } \\ m & \text { number of observations } \\ M & \text { mass of adsorbent } \\ n & \text { fitting parameter of Eq. (19) } \\ N_{\mathrm{o}} & \text { adsorption capacity per unit volume of fixed bed } \\ p & \text { number of fitting parameters } \\ q_{\mathrm{o}} & \text { adsorption capacity per unit mass of adsorbent } \\ Q & \text { volumetric flow rate } \\ R^{2} & \text { coefficient of determination } \\ t & \text { time or number of pore volumes } \\ t_{\mathrm{I}} & \text { time value at inflection point }\end{array}$

$t^{*} \quad$ unit time in the chosen system of units

$u \quad$ superficial velocity

$w_{i} \quad$ Akaike weight for model $i$

$Y \quad$ response variable of the Gompertz equation

$Y_{\mathrm{I}} \quad$ response variable value at inflection point

$Y_{\max } \quad$ maximum $Y$ value

$z_{j}^{\text {mod }} \quad$ model prediction for observation $j$

$\bar{z}^{\exp } \quad$ mean of experimental data

$z_{j}^{e x p} \quad$ experimental data for observation $j$

\section{Greek letters}

$\alpha \quad$ parameter of the Gompertz equation

$\beta \quad$ parameter of the Gompertz equation

$\Delta_{i} \quad$ difference in $\mathrm{AIC}_{\mathrm{c}}$ between two models

$\tau \quad$ time required for $50 \%$ breakthrough

Initialisms

$\mathrm{AIC}_{\mathrm{c}} \quad$ corrected Akaike information criterion

$\mathrm{AIC}_{\mathrm{c}, i} \quad \mathrm{AIC}_{\mathrm{c}}$ value for model $i$

$\mathrm{AIC}_{\mathrm{c}, \min }$ lowest $\mathrm{AIC}_{\mathrm{c}}$ value

SSE sum of squared errors follows that the Bohart-Adams, Thomas, and Yoon-Nelson models all suffer from the same limitation. Thus, it is no surprise that some papers have reported experimental breakthrough curves that were not amenable to treatment by these logistic-based models [13-20]. Such curves were found to display an asymmetric, tailing behavior, i.e., a slow approach of $C / C_{\mathrm{o}}$ toward unity.

Relatively little progress has been made in the development of models that are capable of describing asymmetry. Some investigators have proposed the use of neural network or hybrid Thomas-neural network models in lieu of the Thomas model to fit asymmetric breakthrough curves [16-20]. However, these neural network models are coded in specialized software; they are not available in the form of simple equations that allow testing by other researchers.

This paper reports the use of a simple mathematical expression, known as the Gompertz equation [21], to describe the asymmetric characteristics of fixed bed breakthrough curves. Although the Gompertz equation is in common use across a wide range of disciplines, it has thus far attracted little attention as a modeling tool in environmental adsorption research. Like the logistic equation of Verhulst, Gompertz's equation has been used extensively in the studies of growth processes. It produces sigmoidal curves that describe the acceleration, inflection, and deceleration phases of growth over time.

In the domain of environmental technology research, several modified forms of the Gompertz model have been used to describe microbial growth, substrate consumption, and biogas production rates in waste treatment processes based on anaerobic fermentation [22]. The Gompertz equation has also been used to correlate the breakthrough behavior of fixed bed columns packed with nanomaterials [23-25]. These fixed bed columns were not designed to remove contaminants by adsorption; they were used to disinfect drinking water contaminated by harmful microorganisms.

There are only limited applications of the Gompertz equation in the realm of environmental adsorption research. Yu et al. [26] used a modified Gompertz equation to model variations of a Freundlich isotherm parameter and a surface diffusion coefficient with time. Another modified version of the Gompertz equation was used by Çelekli et al. [27] to model the kinetics of copper adsorption in a batch adsorber. Smith et al. [28] used the Gompertz equation to describe the breakthrough behavior of a rapid small-scale column test apparatus but only one set of breakthrough data was presented in their work. Hu et al. [29] used a double exponential equation, which is similar to the Gompertz equation, to fit two sets of breakthrough data displaying slight asymmetry. The curve-fitting ability of the Gompertz equation was not sufficiently tested in these two prior studies on fixed bed adsorption $[28,29]$. Accordingly, the main aim of this research is to determine how well breakthrough curve asymmetry could be described by the Gompertz equation. The skewed breakthrough data examined here were collated from reports published in the environmental adsorption literature.

\section{Theory}

The British mathematician, Benjamin Gompertz, put forward a model in 1825 [21] as a means to explain human mortality curves. Modern studies have successfully exploited Gompertz's model to describe growth curves across a wide range of disciplines, such as biology, crop science, medical science, engineering, and economics, to name but a few. A commonly used form of the Gompertz equation for growth [30] reads

$Y=Y_{\max } \exp [-\exp (\alpha-\beta t)]$

where $Y$ denotes the response variable at time equal to $t, Y_{\max }$ represents the maximum $Y$ value or upper asymptote, and $\alpha$ and $\beta$ are constants. The three parameters to be fitted are $Y_{\max }, \alpha$, and $\beta$.

The Gompertz equation produces sigmoidal curves of asymmetric shape. A typical feature of these sigmoidal curves is the existence of an inflection point at which the growth rate is maximized. The inflection point and maximum growth rate of a Gompertz curve may be determined from the first and second derivatives of Eq. (8) which are given by Eqs. (9) and (10), respectively.

$\frac{\mathrm{d} Y}{\mathrm{~d} t}=Y_{\max } \beta \exp (\alpha-\beta t) \exp [-\exp (\alpha-\beta t)]$

$\frac{\mathrm{d}^{2} Y}{\mathrm{~d} t^{2}}=Y_{\max } \beta^{2}[\exp (\alpha-\beta t)-1] \exp (\alpha-\beta t) \exp [-\exp (\alpha-\beta t)]$

By setting the left-hand side of Eq. (10) to zero, we obtain the expression for the time value, $t_{\mathrm{I}}$, at the inflection point:

$t_{\mathrm{I}}=\frac{\alpha}{\beta}$ 